\title{
Reactivity in combustion process for expanded graphites: influence of dimensional effect
}

\author{
V. A. Logvinenko ${ }^{1,2}$, V. G. Makotchenko ${ }^{1}$, V.E. Fedorov ${ }^{1,2}$ \\ ${ }^{1}$ Nikolaev Institute of Inorganic Chemistry, Siberian Branch of the Russian Academy \\ of Sciences, Novosibirsk 630090, Russia \\ ${ }^{2}$ Novosibirsk State University, Novosibirsk 630090, Russia \\ val@niic.nsc.ru,mwg@niic.nsc.ru,fed@niic.nsc.ru
}

PACS 65.80.Ck; 65.80g; 82.60.Qr

DOI 10.17586/2220-8054-2016-7-1-234-243

\begin{abstract}
Thermal stability in combustion reaction for natural graphite, graphene and several expanded graphite phases were studied; the kinetic parameters of the oxidation reaction were calculated for two samples. Natural graphite (crystalline particles $200-300 \mu \mathrm{m}$ ) has the maximum stability $\left(E_{1}=201 \pm 2 \mathrm{~kJ} / \mathrm{mol}, \lg A_{1}=7.1 \pm 0.1\right.$ ), while multilayer graphene is the most reactive $\left(E_{2}=120 \pm 1 \mathrm{~kJ} \mathrm{~mol}^{-1}, \lg A_{2}=4.3 \pm 0.10\right)$. The different sample grain sizes and their different structures result in different thermal stabilities: both in the reaction zones location (i.e. in the topochemical equation forms), and in the kinetic parameters' values.
\end{abstract}

Keywords: natural graphite, graphene, expanded graphite, thermal stability, non-isothermal kinetics, combustion.

Received: 20 November 2015

\section{Introduction}

A very noticeable difference is known to exist between a compound's thermodynamic properties for its bulk crystal form versus that of its nanosized particles. For example, the melting point for single nanoparticles of $\mathrm{Au}$ with a mean diameter of $4 \mathrm{~nm}$ is lower than the bulk value by over $400{ }^{\circ} \mathrm{C}$ [1]; the melting temperature of aluminum decreases from $660{ }^{\circ} \mathrm{C}$ to $650{ }^{\circ} \mathrm{C}$ and to $620{ }^{\circ} \mathrm{C}$ respectively for the bulk crystal, $30 \mathrm{~nm}$ and $6 \mathrm{~nm}$ nanoparticles, respectively [2]. Potassium nitrate nanoparticles melt at a lower temperature $\left(313{ }^{\circ} \mathrm{C}\right)$ than the crystal $\left(333{ }^{\circ} \mathrm{C}\right)[3]$.

It was demonstrated that the intense mechanical treatment of coal (i.e. grinding of particles) causes an increase in its reactivity, which is exhibited both as a decrease in the temperature interval of the combustion, and a decrease in the activation energy [4]. The difference in reactivity can be expected for graphite phases consisting of particles with different sizes and structures.

The oxidation of natural graphite was considered in several detailed papers [5-7]. It was shown that for a prismatic graphite structure, the fraction of edge sites increases, and the fraction of basal plane sites decreases, as the particle size decreases. This confirms that the ratio between edge sites and basal sites has a strong influence on all thermal parameters for graphite oxidation [7].

The activation energy for basic graphite or carbon material follows a distribution similar to that of a probability density function and this will be greatly enhanced by the imperfections in the char structures [5].

There are no direct comparisons of natural graphite, expanded graphites and graphene with regards to the ease of their oxidation in the literature. 


\section{Experimental}

\subsection{Synthesis of graphite phases}

Dicarbon polyfluoride with the intercalated chlorine trifluoride was synthesized by the vigorous fluorination of graphite with the liquid chlorine trifluoride using the well-known procedure [8]. Graphitic oxide was synthesized by the oxidation of graphite in concentrated sulfuric acid according to the methods of Hummers and Offeman [9]. The syntheses of graphitic nitrate and graphitic bisulfate were carried out by the electrochemical oxidation of the graphite in of sulfuric or nitric acid media [10-12].

The multilayer graphene (phase I) was produced by the thermal shock-loading at $800-$ $1000{ }^{\circ} \mathrm{C}$. Calculations using the Scherrer equation [13] showed that the graphene stock thickness (along the $c$-axis) was $3 \mathrm{~nm}$. The expanded graphites (phases II, III and IV) were produced from graphitic oxide, nitrate and bisulphate by the thermal shock loading at $800-1000{ }^{\circ} \mathrm{C}$. Scherrer equation calculations showed that the graphene stock thicknesses (along the $c$-axes) were 17,42 and $83 \mathrm{~nm}$, respectively.

The natural graphite (phase V) (from Zavaljevskoje deposit) had particles of 200 $300 \mu \mathrm{m}$ and the ash content was less than $0.1 \mathrm{wt} \%$.

\subsection{Thermal analysis}

The oxidation of the graphite phases was studied in the standard experimental conditions: Netzsch thermal analyzer TG 209 F1, corundum crucible, argon + oxygen mixture flow $(40+$ $10 \mathrm{~cm}^{3} \cdot \mathrm{min}^{-1}$ ), sample masses $\approx 2.0 \mathrm{mg}$. TG curves were obtained with the heating rates of 3 , 5, 10, 20 and $40 \mathrm{~K} / \mathrm{min}$.

\subsection{Kinetic analysis under non-isothermal conditions}

Thermogravimetric data were processed using the computer program Netzsch Thermokinetics 2 (Version 2004.05) [14,15]. A special program module, "Model-free", based on well-known studies [16-25], allows multiple thermogravimetric curves, obtained with different heating rates, to be processed and the activation energy to be calculated without preliminary information about the kinetic topochemical equation. The Friedman method was used to calculate the activation energies for each experimental point of fractional conversion (in the range $0.005<\alpha<0.995)$.

If the activation energy is variable in compliance with the Friedman method, the decomposition process is a multi-step reaction.

We then used the same set of experimental data to search for the corresponding topochemical equation (the selection was made from 16 equations: chemical reaction at the interface, nucleation and diffusion). This calculation was made by the improved differential procedure of Borchardt-Daniels $[14,26]$ within the multiple linear regression approach. It is very important that the range for the degree of conversion $(\alpha)$ for this calculation be chosen based on the relative constancy of the calculated kinetic parameters from the prior Friedman analysis.

The Fisher test [14] was used to search for the best kinetic description and for statistical control of the obtained equation. This tested the residual variance of the individual models against one another and answers the question of whether the models differ significantly (statistically) or not. If $F_{\exp (1)} \approx F_{\exp (2)}$ for two equations, there is no reason to assume the first model is better at characterizing the experiment. The statistical quantile $F_{\text {crit }}$ is obtained for a level of significance of 0.05 . 
If the calculation results in two or three kinetic equations with close values in their correlation coefficients and on the $F$ test, but with noticeably different values in kinetic parameters, it is most correct to choose the equation with activation energy values closest to the data from the "Model-free" module program. Discrimination between the two steps is relative in this search for topochemical equations, but helps to identify the most reliable ones. The special program of nonlinear regression is useful in searching for a full set of kinetic parameters for multi-stage processes. The closest fit between the activation energies from the "Model-free" analysis and the nonlinear regression calculation is important from a physicochemical point of view. Therefore, the authors of the computer program used recommend fixing $E$ values (obtained by linear regression and congruent with $E$ from the "Model-free" analysis) in calculations with this program.

The random error in the activation energy values for such a reversible decomposition reaction is usually about $10 \%$ in these experiments, which we took into consideration. The computer program Netzsch Thermokinetics 2 enables estimation for the contribution of each stage (as $\Delta m$ portion) following this nonlinear regression calculation.

Well-known recommendations for performing kinetic computations on thermal analysis data $[27,28]$ were used; new studies on non-isothermal kinetics were taken into account $[29,30]$.

There were several important assumptions and limitations. The kinetic equations to calculate the kinetic parameters are topochemical and the calculated parameters $(E$ and $A)$ are formal and conventional from the standpoint of the classical chemistry of solids.

However, the general trend in the variation of these values within a specially selected series of compounds (either isostructural or genetically related) is very important because the expected disorder in the reaction zones can be identical for them; all other errors and simplifications will be minimized and smoothed in such a comparison. The best series are coordination compounds with volatile ligands (with one central atom and different ligands or with different central atoms and the same ligand) or inclusion compounds [31-34].

The studied series are several graphite phases with the modified structures; the temperature intervals of the combustion, topochemical equations and the kinetic parameter values can be compared in such series.

\section{Results and Discussion}

Thermogravimetric curves for the combustion of several graphite phases are shown in Fig. 1. So, as the curves' temperature intervals are directly connected with the rate constant of the thermal decomposition processes [31,32], the positioning of curves in the temperature series fully correlates with the kinetic stability. Therefore, the thermal (kinetic) stability of the phases under the oxidation (combustion) increases in the series: $\mathbf{I}<\mathbf{I I}<\mathbf{I I I}<\mathbf{I V}<\mathbf{V}$ (Fig. 1). This fully correlates with the stock thicknesses of the samples $(3,17,42,83 \mathrm{~nm}$ and $200-300 \mu \mathrm{m})$. Two phases, the multilayer graphene and the natural graphite (the leftmost and the rightmost of the series), were chosen for detailed kinetic study.

\subsection{Combustion of multilayer graphene (I)}

Thermogravimetric curves, obtained using heating rates 10,20 and $40 \mathrm{~K} \cdot \mathrm{min}^{-1}$, were used for kinetic analysis (Fig. 2). "Model-free" data are given in Fig. 3. The activation energy can be considered a variable in compliance with the Friedman method; therefore, the decomposition reaction is a multi-step process. The best description of the reaction is a two-step process $(\mathrm{A} \rightarrow \mathrm{B} \rightarrow \mathrm{C}$ ) with an order equation (Fn) and Prout-Tompkins equation (B1) (Table 1).

The most probable estimate is two consecutive reactions (Fig. 4):

$\mathrm{A} \rightarrow$ B. Fn, $f_{1}(\alpha)=(1-\alpha)^{0}, E_{1}=120 \pm 1 \mathrm{~kJ} \mathrm{~mol}^{-1}, \lg A_{1}=4.3 \pm 0.1$. 


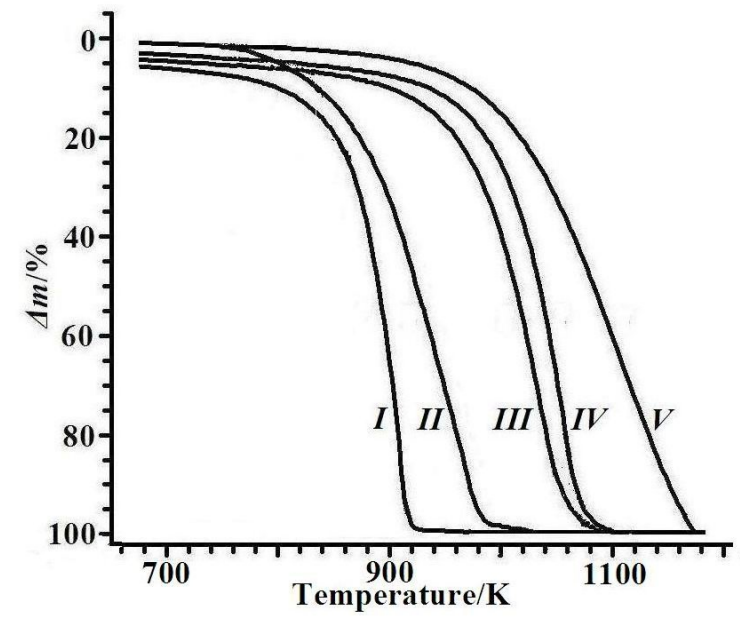

FIG. 1. TG curves of the combustion processes for multilayer graphene (I), the expanded graphite phases (II, III, IV) and natural graphite (V). The heating rate was $10 \mathrm{~K} \cdot \mathrm{min}^{-1}$

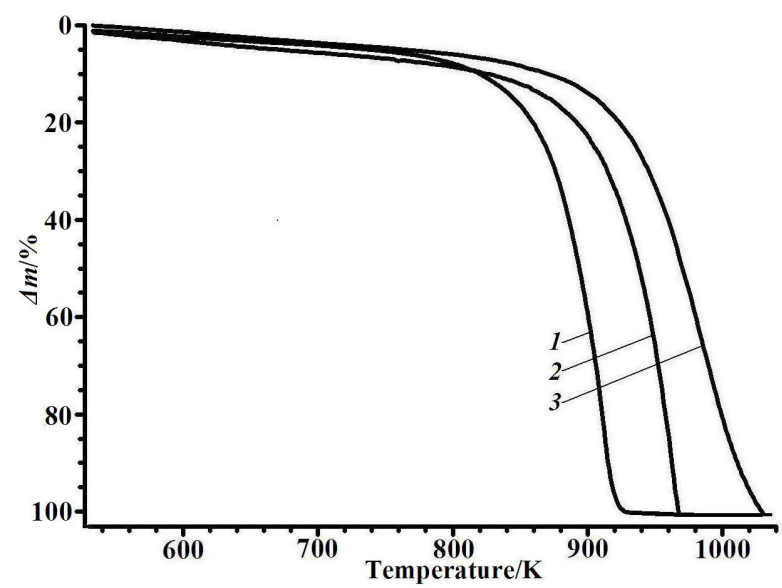

FIG. 2. TG-curves of the combustion process of the multilayer graphene (I). The heating rates were $10(1), 20(2)$ and $40(3) \mathrm{K} \cdot \mathrm{min}^{-1}$

$\mathrm{B} \rightarrow \mathrm{C} . \mathrm{B} 1, f_{2}(\alpha)=\alpha \cdot(1-\alpha), E_{2}=31 \pm 5 \mathrm{~kJ} \mathrm{~mol}^{-1}, \lg A_{2}=0.7 \pm 0.3$.

Corr. coeff. $=0.993421$.

The time dependencies of the yield for each reactant in the decomposition are shown in Fig. 5. The computer program used enables estimation of the contribution of each stage (as $\Delta m$ portion) after the nonlinear regression calculation. Therefore, the first stage $(\mathrm{A} \rightarrow \mathrm{B})$ corresponds to $82 \%$, and the second stage $(\mathrm{B} \rightarrow \mathrm{C})$ corresponds to $18 \%$ of this combustion reaction.

The zero order reaction (i.e. the constant decomposition rate) can be connected with the reaction zone, formed on the grain's surface and moving perpendicularly to the graphene layers. The final oxidation step ( $18 \%$ of the mass loss) was described by the Prout-Tompkins equation $\left(E_{2}=31 \pm 1 \mathrm{~kJ} \cdot \mathrm{mol}^{-1}, \lg A_{2}=0.7 \pm 0.3\right.$ ); this part of the combustion takes place with the reaction acceleration (as the rapid ignition of the particle nuclei). 


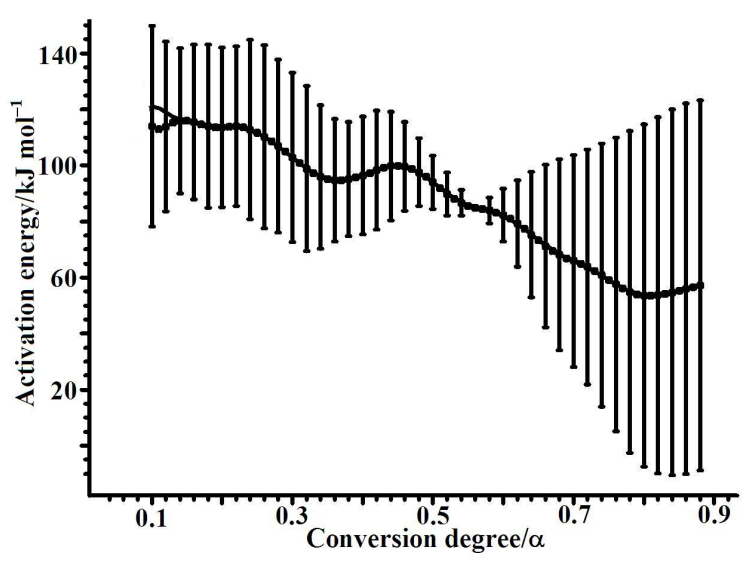

FIG. 3. Friedman analysis of the combustion process of the multilayer graphene (I): activation energies depending on the degree of conversion $\alpha$. Perpendicular lines SD of calculation

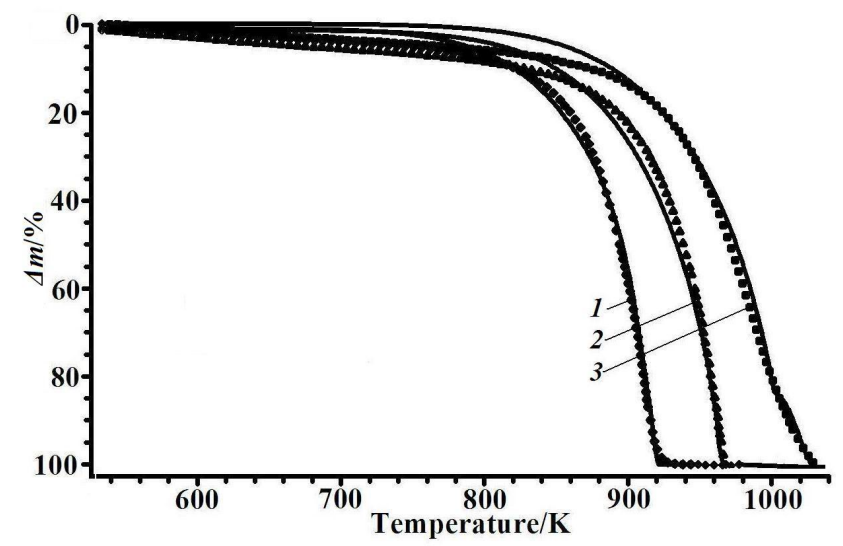

FIG. 4. Data processing for the combustion process of the multilayer graphene (I). TG curve fitting of non-linear regression, simulated with two consecutive reactions (equations Fn and B1). The points are the experimental data; the lines are the calculated data. The heating rates were 10 (1), 20 (2) and 40 (3) $\mathrm{K} \cdot \mathrm{min}^{-1}$

\subsection{Combustion of natural graphite (V)}

Thermogravimetric curves, obtained with the heating rates 3,5 and $10 \mathrm{~K} \cdot \mathrm{min}^{-1}$, were used for kinetic analysis (Fig. 6). "Model-free" data are given in Fig. 7. The activation energy can be considered a variable in compliance with the Friedman method; therefore, the decomposition process is a multi-step reaction (Table 2).

The most probable estimate is two consecutive reactions:

$\mathrm{A} \rightarrow$ B. An, $f_{1}(\alpha)=(1-\alpha) /[-\ln (1-\alpha)]^{0.1}, E_{1}=203 \pm 1 \mathrm{~kJ} \cdot \mathrm{mol}^{-1}, \lg A_{1}=9.7 \pm 0.1$.

$\mathrm{B} \rightarrow$ C. An, $f_{2}(\alpha)=(1-\alpha) /[-\ln (1-\alpha)]^{0.2}, E_{2}=201 \pm 3 \mathrm{~kJ} \cdot \mathrm{mol}^{-1}, \lg A_{2}=7.0 \pm 0.1$.

Corr. coeff. $=0.998606$.

The computer program used enables estimation of the contribution of each stage (as $\Delta m$ portion) after the nonlinear regression calculation. Therefore, the first step $(\mathrm{A} \rightarrow \mathrm{B})$ corresponds to $1 \%$, and the second step $(\mathrm{B} \rightarrow \mathrm{C})$ corresponds to $99 \%$ of this combustion reaction. So as the first step's contribution is negligible, we recalculate the data as a one-step process (Fig. 8): 
TABLE 1. Multilayer graphene combustion. Data on the F-test of fit quality/to identify the best kinetic description

\begin{tabular}{|c|c|c|c|c|}
\hline$F_{\text {crit }}$ & $F_{\text {exp }}$ & $F_{\text {act }}$ & $\begin{array}{c}\text { Equation } \\
\mathrm{A} \rightarrow \mathrm{B}\end{array}$ & $\begin{array}{c}\text { Equation } \\
\mathrm{B} \rightarrow \mathrm{C}\end{array}$ \\
\hline 1.10 & 1.00 & 1103 & $\mathbf{A n}$ & $\mathbf{A n}$ \\
1.10 & 1.05 & 1102 & $\mathbf{A n}$ & $\mathbf{B n a}$ \\
1.10 & 1.77 & 1104 & An & $\mathrm{B} 1$ \\
1.10 & 2.49 & 1102 & Fn & Bna \\
1.10 & 2.49 & 1104 & Fn & B1 \\
1.10 & 2.50 & 1103 & Fn & An \\
1.10 & 2.85 & 1106 & Fn & Fn \\
1.10 & 5.25 & 1107 & An & \\
\hline
\end{tabular}

Notes: $F_{\text {exp }}$ - experimental F-value; $F_{\text {crit }}$ - critical F-value, calculated for the degree of freedom of current model $F_{\text {act }}$ and the degree of freedom of the best model, at the one-sided level of confidence of 0.95. $F_{\text {act }}$ is the degree of freedom (number of data points - number of optimized parameters) of the current model.

The used topochemical equations are Avrami-Erofeev (An), $n$-th order (Fn) and ProutTompkins (B1 and Bna) equations [14-15].

The parameter calculation for An-An, An-Bna, Fn-An, Fn-Fn equations combination includes both one mass loss stage, and one mass increase stage, so these mathematical solutions have no physical-chemical meaning for the mass loss TG-curves.

TABLE 2. Natural graphite combustion. Data on the F-test of fit quality/to identify the best kinetic description

\begin{tabular}{|c|c|c|c|c|}
\hline$F_{\text {crit }}$ & $F_{\text {exp }}$ & $F_{\text {act }}$ & $\begin{array}{c}\text { Equation } \\
\mathrm{A} \rightarrow \mathrm{B}\end{array}$ & $\begin{array}{c}\text { Equation } \\
\mathrm{B} \rightarrow \mathrm{C}\end{array}$ \\
\hline 1.10 & 1.00 & 1174 & $\mathrm{An}$ & $\mathrm{An}$ \\
1.10 & 1.05 & 1178 & $\mathrm{An}$ & \\
1.10 & 1.41 & 1178 & $\mathrm{Fn}$ & \\
1.10 & 1.45 & 1178 & $\mathrm{C} 1 \mathrm{~B}$ & \\
1.10 & 2.36 & 1179 & $\mathrm{D} 3$ & \\
1.10 & 71.02 & 1179 & $\mathrm{~B} 1$ & \\
\hline
\end{tabular}

Notes: $F_{\text {exp }}$ - experimental $F$-value; $F_{\text {crit }}-$ critical $F$-value, calculated for the degree of freedom of current model $F_{\text {act }}$ and the degree of freedom of the best model, at the one-sided level of confidence of 0.95. $F_{\text {act }}$ is the degree of freedom (number of data points - number of optimized parameters) of the current model.

The used topochemical equations are Avrami-Erofeev (An), $n$-th order (Fn) and ProutTompkins (B1) equations [14-15]. 


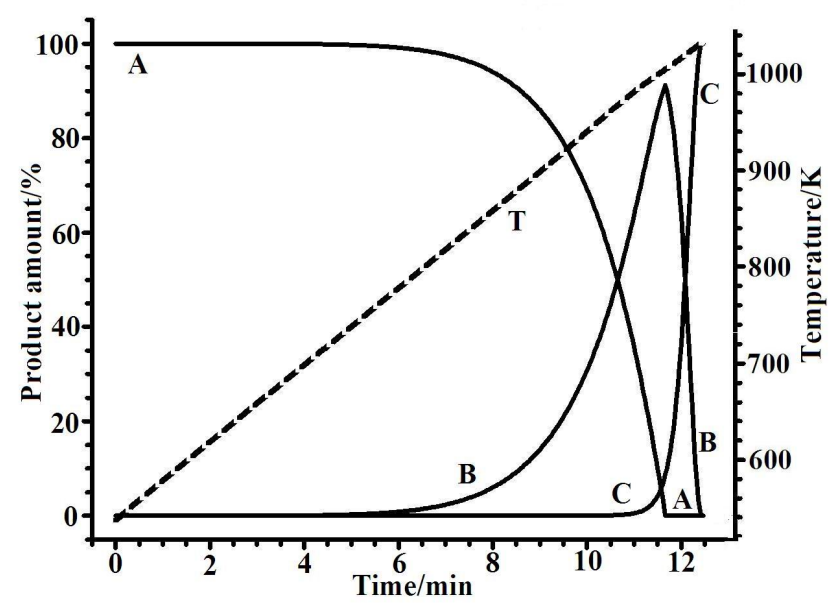

FIG. 5. The combustion process of the multilayer graphene. Time dependence of the yield of each reactant in the decomposition. The calculation corresponds to two-stage consecutive reactions $(\mathrm{A} \rightarrow \mathrm{B} \rightarrow \mathrm{C})$ in Fig. 4. The heating rate was $40 \mathrm{~K} \cdot \mathrm{min}^{-1}$

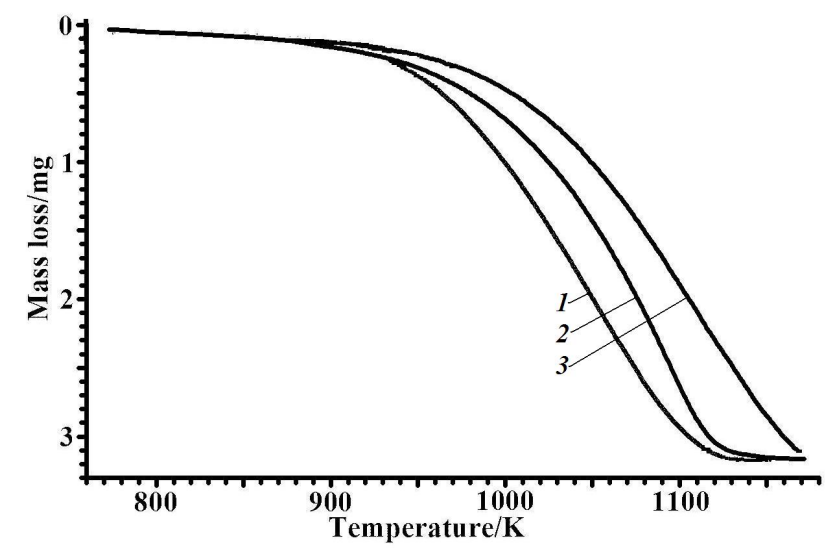

FIG. 6. TG-curves of the combustion process of the natural graphite sample (V). The heating rates were $3(1), 5(2)$ and $10(3) \mathrm{K} \cdot \mathrm{min}^{-1}$

$\mathrm{A} \rightarrow$ B. An, $f(\alpha)=(1-\alpha) /[-\ln (1-\alpha)]^{0.2}, E=201 \pm 2 \mathrm{kJmol}^{-1}, \lg A_{2}=7.1 \pm 0.1$. Corr. coeff. $=0.999120$.

Avrami-Erofeev topochemical equation denotes the evident diffusion contribution.

The calculated activation energy stays within the known broad averaged value: $E=$ $167.5 \pm 50 \mathrm{~kJ} \cdot \mathrm{mol}^{-1}$ for natural graphite oxidation $[5,6]$. Such a broad estimation of the activation energy can be explained by the different properties of natural graphite samples from different locations.

\section{Conclusion}

The different sizes of sample particles and their different structures result in different thermal stabilities: both in the reaction zones location (i.e. in the topochemical equation forms), and in the values of kinetic parameters. 


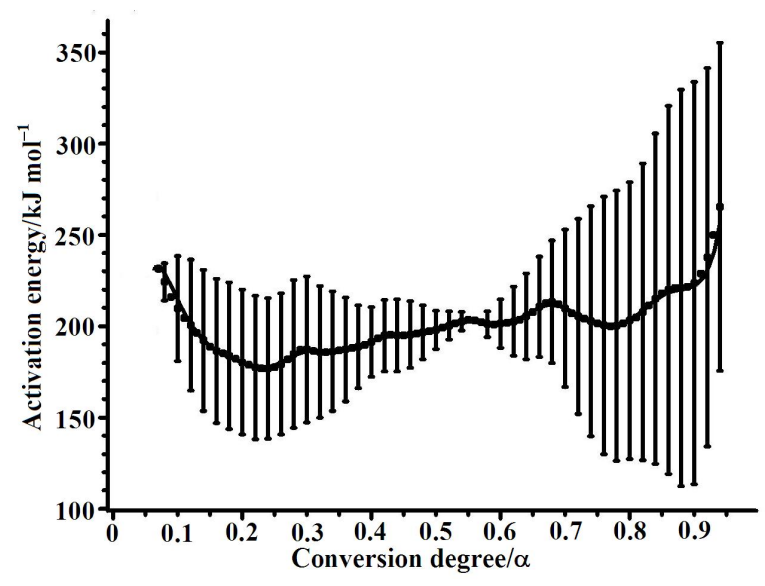

FIG. 7. Friedman analysis of the combustion process of the natural graphite sample (V): activation energies depending on the degree of conversion $\alpha$. Perpendicular lines SD of calculation

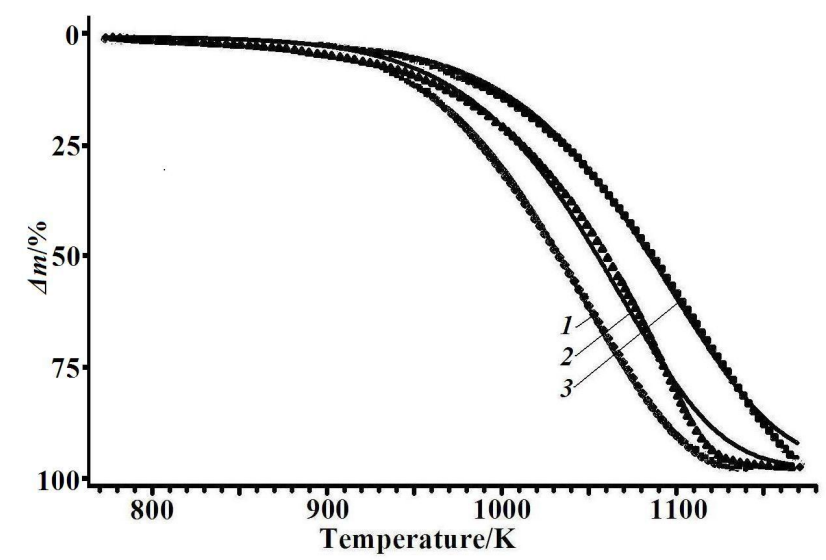

FIG. 8. Data processing for the combustion process of the natural graphite sample $(\mathbf{V})$. TG curve fitting of non-linear regression, simulated with the single stage reaction (equation An). The points are the experimental data; the lines are the calculated data. The heating rates were $3(1), 5(2)$ and $10(3) \mathrm{K} \cdot \mathrm{min}^{-1}$

Graphite is a very anisotropic layered substance. For an ideal single crystal, the ratio of the reaction rates on the different crystal faces must reach a factor of $10^{15}$ times, but for the real graphite single crystal, this ratio is reduced to only $10^{2}$ [35]. This fact can be connected to the reaction zone composition. The actual structure of the crystal surface is disordered; the bond lengths are increased, and the reaction zone formed on different crystal faces is rather uniformly arranged.

Therefore, we can expect the close-ratio kinetic parameters for different crystal faces [31], even through such anisotropic crystals of graphite particles. The calculated kinetic parameters for the natural graphite sample stay fairly close to the known averaged value [56].

The flat (quasi two-dimensional) morphology of the multilayer graphene particles exhibits the peculiarity in the kinetic topochemistry: the zero order reaction (which is the constant rate of the combustion). 
We think that the formal kinetic approach used does not give one the possibility to discuss the difference in the kinetic stability based on the distribution of basal and edge active sites with different energies and of the formation of semiquinone and epoxy groups as the reaction progresses $[5,7]$.

The thermal stability of the phases under the oxidation (combustion) increases in the series: I $<$ II $<$ III $<$ IV $<\mathbf{V}$. This demonstrates the direct influence of the dimensional effect on the kinetic stability in the combustion chemical reaction.

It is worth noting that this generalized series of thermal stabilities is loosely based on the constant rates, but such a series is not the same as for that of the activation energy values. For the extreme terms: $E_{I}<E_{V}$ actually, the stability level for the expanded graphites (II, III and IV) can be connected with the entropic contribution (e.g. with the pre-exponential factor value).

\section{Acknowledgements}

The work was supported by the Russian Scientific Foundation, Project 14-13-00674.

\section{References}

[1] Buffat Ph., Borel J.P. Size effect on the melting temperature of gold particles. Phys. Rev., 1976, A 13, P. 2287-2298.

[2] Lai S.L., Carlsson J.R.A., Allen L.H. Melting point depression of Al clusters generated during the early stages of film growth: Nanocalorimetry measurements. Appl. Phys. Lett., 1998, 72, P. 1098-1100.

[3] Bulavchenko A.I., Demidova M.G., Podlipskaya T.Yu., Tatarchuk V.V., Druzhinina I.A. Alekseev A.V., Logvinenko V.A., Drebushchak V.A. Microemulstion synthesis of powders of water soluble energy saturated salts. Russ. J. Inorg. Chem., 2012, 57(6), P. 769-776.

[4] Yusupov T.S., Shumskaya L.G., Burdukov A.P., Logvinenko V.A. Reactivity of coal of different stages of metamorphism in the processes of thermo-oxidative destruction. Chemistry for Sustainable Development, 2011, 19, P. 389-394.

[5] Backreedy R., Jones J.M., Pourkashanian M., Williams A. A study of the reaction of oxygen with graphite : Model chemistry. Faraday Discuss., 2001, 119, P. 385-394.

[6] Bews I.M., Hayhurst A.N., Richardson S.M, Taylor S.G. The order, Arrhenius parameters, and mechanism of the reaction between gaseous oxygen and solid carbon. Combust. Flame, 2001, 124, P. 231-245.

[7] Jianga W., Nadeaub G., Zaghibb K., Kinoshitaa K. Thermal analysis of the oxidation of natural graphite effect of particle size. Thermoch. Acta, 2000, 351, P. 85-93.

[8] Makotchenko V.G., Grayfer E.D., Nazarov A.S., Kim S.-J., Fedorov V.E. The synthesis and properties of highly exfoliated graphites from fluorinated graphite intercalation compounds. Carbon, 2011, 49(10), P. 32333241 .

[9] Hummers Jr.W.S., Offeman R.E. Preparation of graphitic oxide. J. Am. Chem. Soc., 1958, 80(6), P. 1339.

[10] Rudorff W., Hofmann U. Uber Graphitsaltse. Z. Anorg. Allg. Chem. B, 1938, 238(1), P. 1-50.

[11] Metrot A., Fisher J.E. Charge-transfer reactions during anodic oxidation of graphite in $\mathrm{H}_{2} \mathrm{SO}_{4}$. Synth. Met., 1981, 3, P. 201-207.

[12] Avdeev V.V., Monyakina L.A., Nikolskaya I.V., Sorokina N.E., Semenenko K.N. The choice of oxidizers for graphite hydrogen sulfate chemical synthesis. Carbon, 1992, 30(6), P. 819-824.

[13] Scherrer P. Bestimmung der Grösse und der Inneren Struktur von Kolloidteilchen Mittels Röntgenstrahlen, Nachrichten von der Gesellschaft der Wissenschaften, Göttingen. Mathematisch-Physikalische Klasse, 1918, 2, P. $98-100$.

[14] Netzsch Thermokinetics. http:/www.netzsch-thermal-analysis.com/us/products-\%20\%20solutions/ advancedsoftware/thermokinetics.html

[15] Moukhina E. Determination of kinetic mechanisms for reactions measured with thermoanalytical instruments. J. Therm. Anal. Calorim., 2012, 109, P. 1203-1214.

[16] Kissinger H.E. Reaction kinetics in differential thermal analysis. Anal. Chem., 1957, 29, P. $1702-1706$.

[17] Friedman H.L. Kinetics of thermal degradation of char-forming plastics from thermogravimetry. J. Polym. Sci., 1963, 6, P. 183-195. 
[18] Ozawa T. A new method of analyzing thermogravimetric data. Bull. Chem. Soc. Japan, 1965, 38, P. 18811886.

[19] Ozawa T. Estimation of activation energy by isoconversion methods. Thermochim. Acta, 1992, 203, P. 159165.

[20] Flynn J.H., Wall L.A. General treatment of the thermogravimetry of polymers. J. Res. Nat. Bur. Stand., 1966, 70, P. 478-523.

[21] Opfermann J., Kaisersberger E. An advantageous variant of the Ozawa-Flynn-Wall analysis. Thermochim. Acta, 1992, 203, P. 167-175.

[22] Opfermann J.R., Kaisersberger E., Flammersheim H.J. Model-free analysis of thermo-analytical data - advantages and limitations. Thermochim. Acta, 2002, 391, P. 119-127.

[23] Vyazovkin S. Model-free kinetics: staying free of multiplying entities without necessity. J. Therm. Anal. Calorim., 2006, 83, P. 45-51.

[24] Simon P. Single-step kinetics approximation employing non-Arrhenius temperature functions. J. Therm. Anal. Calorim., 2005, 79, P. 703-708.

[25] Simon P. The single-step approximation: attributes, strong and weak sides. J. Therm. Anal. Calorim., 2007, 88, P. 709-715.

[26] Borchard H.J., Daniels F. The application of differential thermal analysis to the study of reaction kinetics. $J$. Amer. Chem. Soc., 1957, 79, P. 41-46.

[27] Vyazovkin S., Burnham A.K., Criado J.M., Luis A., Perez-Maqueda L.A., Popescu C., Sbirrazzuoli N. ICTAC Kinetics Committee recommendations for performing kinetic computations on thermal analysis data. Thermochim. Acta, 2011, 520, P. 1-19.

[28] Vyazovkin S., Chrissafis K., Di Lorenzo M.L., Koga N., Pijolat M., Roduit B., Sbirrazzuoli N., Suñol J.J. ICTAC Kinetics Committee recommendations for collecting experimental thermal analysis data for kinetic computations. Thermochim. Acta, 2014, 590, P. 1-23.

[29] Simon P., Thomas P., Dubaj T., Cibulkova Z., Peller A., Veverka M. The mathematical incorrectness of the integral isoconversional methods in case of variable activation energy and the consequences. J. Therm. Anal. Calorim., 2014, 115, P. 853-859.

[30] Sestak J. Is the original Kissinger equation obsolete today: not obsolete the entire non-isothermal kinetics? $J$. Therm. Anal. Calorim., 2014, 117, P. 3-7.

[31] Logvinenko V. Stability and reactivity of coordination and inclusion compounds in the reversible processes of thermal dissociation. Thermochim. Acta, 1999, 340-341, P. 293-299.

[32] Logvinenko V. Solid state coordination chemistry. The quantitative thermoanalytical study of thermal dissociation reactions. J. Therm. Anal. Calorim., 2000, 60, P. 9-15.

[33] Logvinenko V. Stability of supramolecular compounds under heating. Thermodynamic and kinetic aspects. $J$. Therm. Anal. Calorim., 2010, 101, P. 577-583.

[34] Logvinenko V., Drebushchak V., Pinakov D., Chekhova G. Thermodynamic and kinetic stability of inclusion compounds under heating. J. Therm. Anal. Calorim., 2007, 90, P. 23-30.

[35] Ginak A.Y., Dimshits V.A., Rosovskiy A.Ya. Kinetics of the anisotropic reactions. Part I. Theoretical model. Kinetika i katalis, 1989, 30, P. 83-91 (in Russian). 